\title{
Estatinas: uma revisão baseada na evidência dos efeitos na função cognitiva
}

Tânia Gonçalves*

\section{RESUMO}

Objetivo: O consumo de estatinas tem aumentado ao longo dos anos, tendo sido descritas alterações na função cognitiva. Sabe-se que o cérebro contém cerca de $25 \%$ do colesterol presente no corpo humano. O objetivo desta revisão é avaliar os efeitos laterais cognitivos das estatinas em adultos que tomam esta classe de antidislipidémicos.

Fontes de dados: National Guideline Clearinghouse, Guidelines Finder da National Electronic Library for Health do NHS britânico, Canadian Medical Association Practice Guidelines Infobase, The Cochrane Library, Bandolier, DARE, Clinical Evidence e PubMed.

Métodos de revisão: Pesquisa de artigos publicados entre janeiro de 2013 e janeiro de 2018, nas línguas portuguesa e inglesa, utilizando os termos MeSH Hydroxymethylglutaryl-CoA Reductase Inhibitors e Cognitive Dysfunction. Utilizou-se a escala Strength of Recommendation Taxonomy (SORT), da American Academy of Family Physicians, para atribuição dos níveis de evidência e forças de recomendação.

Resultados: Foram encontrados 58 artigos, dos quais oito cumpriam os critérios de inclusão e exclusão: duas revisões sistemáticas, quatro revisões, um estudo observacional e um artigo de opinião. Após leitura destes estudos verificaram-se resultados contraditórios: uns mostram efeitos laterais das estatinas na cognição, outros não mostram qualquer diferença quando comparados com o placebo e outros ainda sugerem em efeito protetor na demência. Vários estudos não apresentam poder estatístico para avaliar os riscos.

Conclusões: São necessários estudos prospetivos controlados para avaliar os efeitos das estatinas na função cognitiva a curto e a longo prazo. A evidência não apoia a alteração das guidelines, mas defende que as queixas não sejam desvalorizadas.

Palavras-chave: Inibidores de hidroximetilglutaril-CoA redutases; Disfunção cognitiva.

\section{INTRODUÇÃO}

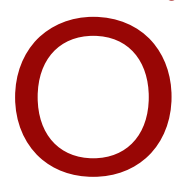

consumo de estatinas em Portugal mantém uma tendência crescente ao longo dos últimos anos. Em 2017 foram comparticipadas e dispensadas, em regime de ambulatório, 9.547.102 embalagens destes fármacos. A sinvastatina costumava ser a estatina mais prescrita, tendo sido substituída pela atorvastatina em 2017. ${ }^{* 1} \mathrm{Em}$ Portugal, estão atualmente comercializados sete fármacos desta classe: atorvastatina, fluvastatina, lovastatina, pitavastatina, pravastatina, rosuvastatina e sinvastatina. $^{2}$

*Interna de Medicina Geral e Familiar, na USF S. João de Braga - ACeS Cávado I.
Têm sido descritas alterações na função cognitiva em utilizadores de estatinas, ${ }^{3}$ estando estas igualmente relatadas no resumo das características do medicamento como efeitos adversos raros e reversíveis com a suspensão destes fármacos. ${ }^{4}$

No que diz respeito à constituição do cérebro humano, sabe-se que este contém $25 \%$ do colesterol presente no corpo humano, sendo este um constituinte essencial das células nervosas e vital para a sua função. ${ }^{5}$

O objetivo desta revisão é avaliar os efeitos laterais das estatinas na cognição nos indivíduos sob terapêutica com esta classe de antidislipidémicos.

*Os dados, solicitados a pedido ao INFARMED, referem-se aos medicamentos comparticipados e dispensados em regime de ambulatório aos utentes do Serviço Nacional de Saúde e subsistemas públicos, no período de 1 de janeiro 2015 a 31 de dezembro de 2017, em Portugal Continental. 


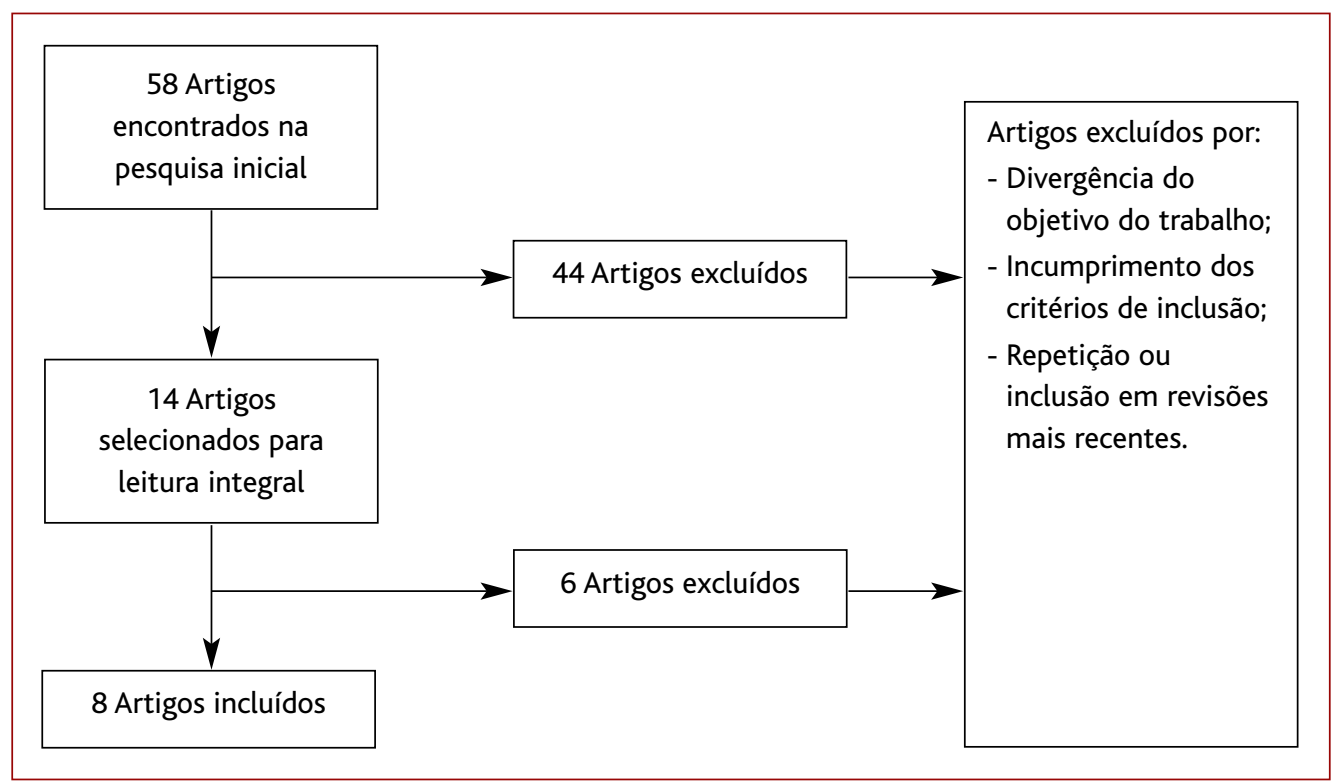

Figura 1. Esquema de seleção dos artigos.

\section{MÉTODOS}

Foi realizada uma pesquisa bibliográfica em janeiro de 2018, utilizando os termos MeSH (Medical Subject Headings) Hydroxymethylglutaryl-CoA Reductase Inhibitors e Cognitive Dysfunction.

Foi selecionada a pesquisa de artigos publicados nas bases de dados National Guideline Clearinghouse, Guidelines Finder da National Electronic Library for Health do NHS britânico, Canadian Medical Association Practice Guidelines Infobase, The Cochrane Library, Bandolier, DARE, Clinical Evidence e PubMed, entre 2013 e 2018, nas línguas portuguesa e inglesa.

A população incluiu indivíduos medicados com estatinas, com idade superior a 18 anos. A variável estudada foi a toma de estatinas e o outcome as alterações da função cognitiva.

Foram excluídos estudos cuja população já apresentasse patologia neurológica (demência, esclerose múltipla, alterações cognitivas ligeiras, doença cerebrovascular), psiquiátrica ou doenças graves (pós bypass coronário, diabetes e doença vascular, sob ventilação mecânica ou cuidados intensivos, sepsia, em quimioterapia, síndroma de Down, bypass cardiopulmonar, pós lesão cerebral traumática grave, pós endarterectomia carotídea), bem como os estudos repetidos.
Utilizou-se a escala Strength of Recommendation Taxonomy (SORT), da American Academy of Family Physicians, para atribuição dos níveis de evidência e forças de recomendação.

\section{RESULTADOS}

Foram encontrados 58 artigos na pesquisa realizada. Destes foram selecionados 14 artigos para leitura integral: duas meta-análises (MA), quatro revisões sistemáticas (RS), cinco revisões, um estudo observacional, um relato de caso e um artigo de opinião. Foram excluídos 44 artigos: 38 por divergirem do objetivo do trabalho ou não cumprirem os critérios de inclusão e seis por serem repetidos. Após leitura integral foram excluídos mais seis artigos: duas MA, três RS e um relato de caso (Figura 1).

Os oito artigos incluídos (duas RS, quatro revisões, um estudo observacional e um artigo de opinião) encontramse descritos na Tabela I. No caso das revisões foram descritos apenas os estudos incluídos nestas que se aproximavam dos objetivos e critérios de inclusão da presente revisão, sendo excluídos os restantes, quer pela descrição do mesmo quer pelo título da respetiva referência.

O primeiro estudo descrito ${ }^{6}$ trata-se da revisão mais recente que inclui três MA, dois relatos de caso e dois estudos prospetivos. A primeira $\mathrm{MA}^{7}$ incluiu 16 estudos 


\begin{tabular}{|c|c|c|c|}
\hline Referência & Estudos/População & Intervenção & Resultados \\
\hline $\begin{array}{l}\text { Mancini et al. } \\
(2016)^{8} \\
\text { RS }\end{array}$ & $\begin{array}{l}20 \text { estudos: } 4 \text { MA, } 1 \text { RS e } 15 \\
\text { estudos observacionais }\end{array}$ & Estatinas & $\begin{array}{l}\text { Estudos observacionais (3) não mostram efeitos } \\
\text { adversos; estudos de caso-controlo, de coorte e } \\
\text { meta-análises sugerem possível benefício (9); (4) } \\
\text { RS e MA não mostram benefício nem efeitos } \\
\text { adversos; estudos de coorte isolados sugerem } \\
\text { diminuição da memória visual (1) em mulheres e } \\
\text { perda de memória após um mês de exposição (1); } \\
\text { uma revisão não mostra associação (1). }\end{array}$ \\
\hline
\end{tabular}

qualitativos e 11 quantitativos em adultos sem história de disfunção cognitiva. Na cognição a curto prazo, avaliada em três ensaios clínicos randomizados (ECR), não se verificaram efeitos adversos (diferença média 1,65; IC95\%, -0,03 a 3,32); na cognição a longo prazo, avaliada em oito ECR e estudos de coorte prospetivos de alta qualidade, três estudos não mostraram associação e cinco estudos mostraram efeito protetor de demência. A síntese quantitativa mostrou redução de $29 \%$ de demência nos utilizadores de estatinas ( $\mathrm{HR}=0,71$; IC95\%, 0,61-0,82). Cinco estudos com follow-up médio de 6,2 anos mostraram uma redução absoluta de $2 \%$ (IC95\%, 1\%-3\%). A segunda MA incluiu 57 estudos (incluindo três ECR, dezasseis estudos de coorte, quatro 


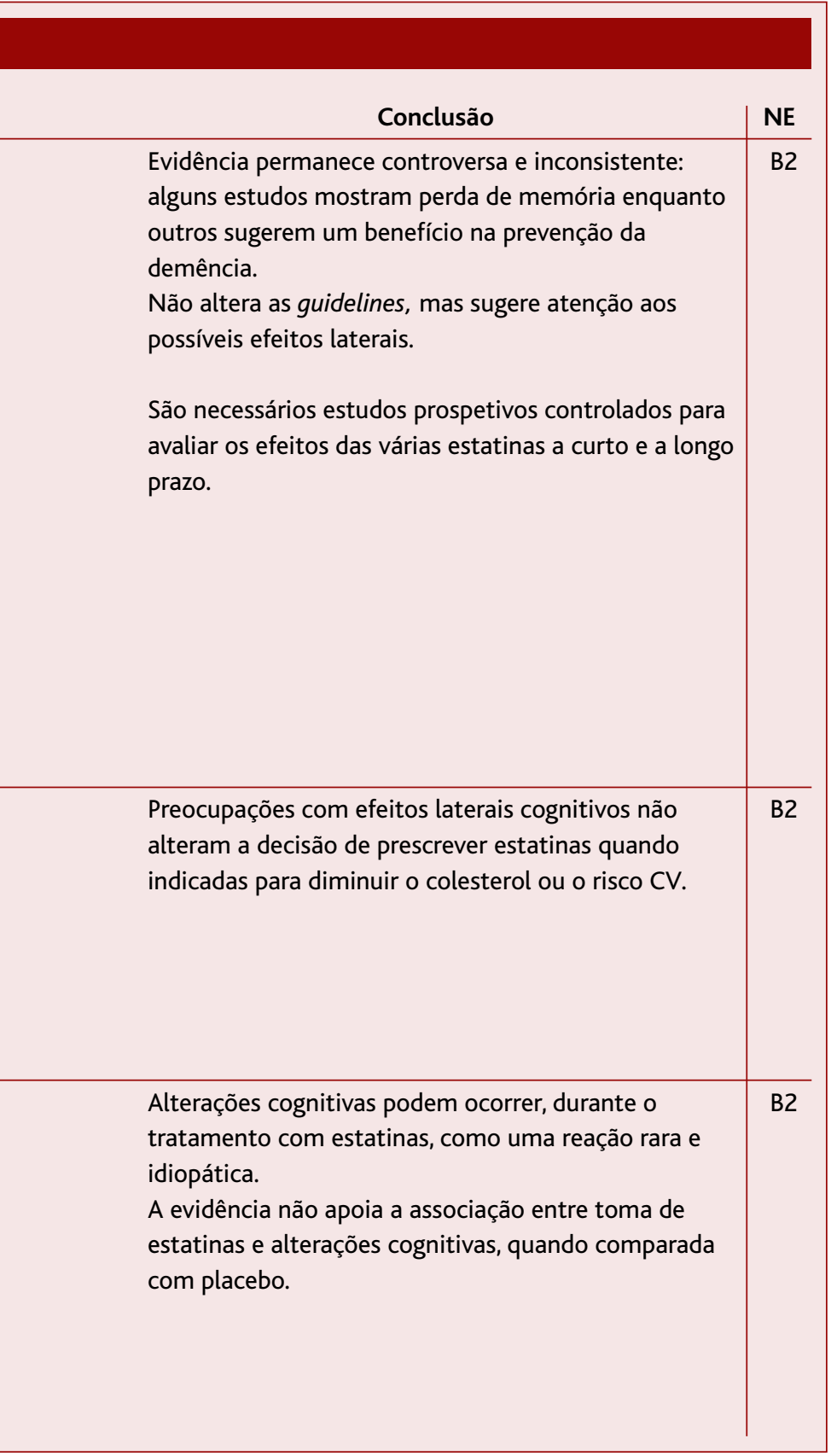

caso-controlo e quatro transversais de qualidade baixa a moderada) e a terceira MA incluiu 23 ECR. Ambas mostraram não haver efeitos estatisticamente significativos na cognição. Estão também incluídos dois relatos de caso, um deles referindo alterações na função cognitiva, relacionadas com a potência da estatina e outro referindo resultados conflituantes. Em ambos os estudos prospetivos mencionados, cada um deles com mais de 2.000 participantes com idade igual ou superior a 65 anos, não houve aumento da incidência de demência, não havendo efeito significativo na diminuição da mesma no primeiro e, por sua vez, parecendo haver um efeito protetor no segundo.

Uma revisão sistemática de 2016 apresenta resultados inconsistentes. ${ }^{8}$ A associação entre estatinas e disfunção cognitiva foi sugerida em estudos de coorte isolados, sendo necessários outros estudos para corroborar estes dados. Assim, as guidelines canadianas concluem que, à luz da evidência atual, não se pode inferir uma verdadeira associação entre o uso de estatinas e disfunção cognitiva, mantendo-se as indicações para a sua prescrição.

Uma revisão de 2016 incluiu quatro MA (três já descritas na revisão de 2017$)^{6}$ e uma RS. ${ }^{9}$ A MA restante ${ }^{10}$ relata que o uso de estatinas foi associado a menor risco de demência e alterações cognitivas, efeito que foi atenuado na análise restrita a estudos de alta qualidade (OR=0,74; IC95\%, 0,62-0,87). A RS incluída no estudo englobou cerca de 26.000 participantes, sendo que cerca de $44 \%$ dessa amostra tinha idade igual ou superior a 70 anos, não se demonstrando alterações cognitivas pelo efeito das estatinas $(0,3 \%$ tanto no grupo das estatinas como no do placebo).

Uma RS de $2015^{11}$ resumiu três MA já descritas nas revisões de 2016 e 2017; uma $\mathrm{RS}^{12}$ que relata que, apesar dos efeitos laterais cognitivos poderem ocorrer a nível individual, a evidência que apoia uma relação causal é fraca ou inexistente, sendo necessários mais estudos; uma revisão, na qual a maioria da investigação clínica não mostra diferenças na alteração da função cognitiva entre estatinas e placebo mas, ainda assim, não exclui a possibilidade de ocorrência deste efeito raro a nível individual; um ECR, no qual a diminuição da função cognitiva e perda de memória ou confusão, ao longo do tempo, foram semelhantes com pravastatina ou placebo; e, por fim, um estudo observacional que admite um efeito protetor das estatinas na demência e disfunção cognitiva.

Numa revisão de 2014, seis ECR não encontraram diferenças entre estatinas e placebo, três ECR mostraram melhorar a função cognitiva com estatinas e apenas um estudo em pacientes com hipercolesterolemia mostrou disfunção cognitiva com a sinvastatina. ${ }^{13} \mathrm{Os}$ 


\section{TABELA I. Estudos incluídos na presente revisão (continuação)}

\begin{tabular}{|c|c|c|c|}
\hline Referência & Estudos/População & Intervenção & Resultados \\
\hline $\begin{array}{l}\text { Šimić et al. } \\
(2015)^{11} \\
\text { RS }\end{array}$ & $\begin{array}{l}7 \text { estudos: } \\
3 \text { MA (já descritas) } \\
1 \text { RS: } \\
\text { - Evidência de qualidade baixa a } \\
{\text { moderada }{ }^{12}}^{1} \\
1 \text { Revisão: } \\
1 \text { ECR: } \\
n=5.804 \text {, seguimento: } 42 \text { meses, } \\
\text { com avaliações a cada } 9 \text { meses. } \\
1 \text { estudo observacional }\end{array}$ & $\begin{array}{l}\text { Estatinas vs } \\
\text { placebo } \\
\text { Estatinas } \\
\text { Estatina vs } \\
\text { placebo } \\
\text { Pravastatina vs } \\
\text { placebo } \\
\text { Estatinas }\end{array}$ & $\begin{array}{l}\text { O risco de efeitos laterais é baixo comparado com } \\
\text { os benefícios das estatinas na prevenção } \\
\text { secundários de eventos CV major. } \\
\text { Sem efeito a curto prazo e possível efeito protetor } \\
\text { a longo prazo. Não apoia alteração das atuais } \\
\text { guidelines. } \\
\text { Sem efeitos na cognição. } \\
\text { Efeitos cognitivos raros, a nível individual, sem } \\
\text { relação causal provada. As queixas dos pacientes } \\
\text { devem ser levadas em consideração e avaliadas } \\
\text { adequadamente. } \\
\text { Maioria não mostra alterações. Possíveis efeitos } \\
\text { raros a nível individual. } \\
\text { Sem diminuição da função cognitiva, perda de } \\
\text { memória ou confusão, ao longo do tempo. } \\
\text { Estatinas podem estar associadas a menor risco de } \\
\text { demência e disfunção cognitiva. }\end{array}$ \\
\hline $\begin{array}{l}\text { Tuccori et al. } \\
(2014)^{13} \\
\text { Revisão }\end{array}$ & $\begin{array}{l}10 \text { ECR } \\
10 \text { Estudos observacionais } \\
8 \text { Relatos de caso }\end{array}$ & $\begin{array}{l}\text { Estatinas vs } \\
\text { placebo } \\
\text { Estatinas: } \\
\text { sinvastatina, } \\
\text { atorvastatina e } \\
\text { rosuvastatina }\end{array}$ & $\begin{array}{l}\text { Resultados contraditórios: } 6 \text { ECR não mostraram } \\
\text { efeito; } 3 \text { demonstraram melhoria; } 1 \text { demonstrou } \\
\text { possível relação entre sinvastatina e disfunção } \\
\text { cognitiva. } \\
\text { Sem efeito na função cognitiva, } 2 \text { mostraram } \\
\text { efeito protetor na demência. } \\
\text { Disfunção cognitiva e alterações da memória } \\
\text { (memória, confusão, concentração). }\end{array}$ \\
\hline $\begin{array}{l}\text { Mandas et al. } \\
(2014)^{14} \\
\text { Estudo } \\
\text { observacional }\end{array}$ & $\begin{array}{l}329 \text { idosos ( } \geq 65 \text { anos), } 137 \text { homens } \\
\text { e } 192 \text { mulheres, ambulatório } \\
\text { (Assemini, Itália) }\end{array}$ & $\begin{array}{l}\text { Toma de } \\
\text { estatinas } \\
\geq 3 \text { anos }\end{array}$ & $\begin{array}{l}\text { Toma de estatinas associada a risco } 2,4 \text { superior de } \\
\text { MMSE anormal }(<24) \text {. }\end{array}$ \\
\hline $\begin{array}{l}\text { McDonagh } \\
(2014)^{15} \\
\text { Artigo de opinião }\end{array}$ & Autorrelato & $\begin{array}{l}\text { Sinvastatina e } \\
\text { pravastatina }\end{array}$ & $\begin{array}{l}\text { Alterações cognitivas com sinvastatina. } \\
\text { Reincidência de sintomas com pravastatina e } \\
\text { desaparecimento de sintomas com a suspensão } \\
\text { dos fármacos. }\end{array}$ \\
\hline $\begin{array}{l}\text { Mancini et al. } \\
(2013)^{16} \\
\text { Revisão }\end{array}$ & $\begin{array}{l}6 \text { estudos: } \\
1 \text { MA } \\
3 \text { revisões } \\
2 \text { ECR }\end{array}$ & $\begin{array}{l}\text { Estatinas } \\
\text { Estatinas } \\
\text { Lovastatina } \\
\text { Sinvastatina }\end{array}$ & $\begin{array}{l}\text { Sem alterações cognitivas. } \\
\text { Efeitos raros, evidência insuficiente para provar } \\
\text { causalidade. Sem associação com tipo ou dose de } \\
\text { estatina, mas sugere-se diferença entre estatinas } \\
\text { hidrofílicas e lipofílicas. } \\
\text { Sem alterações cognitivas com a lovastatina. } \\
\text { Diminuição da função cognitiva com a sinvastatina. }\end{array}$ \\
\hline
\end{tabular}

Legenda: $\mathrm{CV}=$ Cardiovascular; $\mathrm{ECR}=$ Ensaio clínico randomizado; $\mathrm{MA}=$ Meta-análise; MMSE = Mini-Mental State Examination; NE B2 = Evidência inconsistente ou orientada para o paciente de qualidade limitada; NE C3 = Outra evidência baseada em artigo de opinião; RS = Revisão sistemática. 


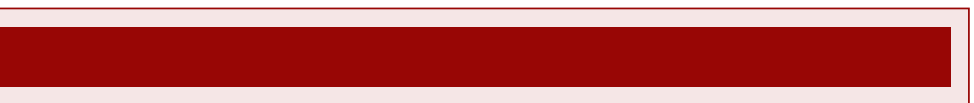
efeitos laterais cognitivos e estudos recentes sugerem um possível efeito protetor na prevenção da demência. ão se prova associação entre o uso de estatinas e

Alterações cognitivas são raras e resolvem-se com a B2 suspensão do fármaco. Estudos com população pequena, sem poder estatístico para avaliar os riscos. São necessários mais estudos.

Estatinas podem ser um fator de risco independente para alterações cognitivas no idoso.

Não desvalorizar queixas de alterações cognitivas.

A evidência disponível não mostra alterações estatisticamente significativas na função cognitiva. estudos observacionais não mostraram associação entre uso de estatinas e alterações cognitivas e dois deles mostraram uma menor prevalência de demência. A idade avançada e as doenças cardiovasculares são possíveis fatores de confundimento a considerar. No que respeita aos relatos de caso foram reportados 19 casos de amnésia potencialmente relacionados com o uso de estatinas e 171 casos de alterações na memória ou disfunção cognitiva. Foram igualmente reportados casos de disfunção cognitiva com o uso de sinvastatina, atorvastatina e rosuvastatina. A idade média foi de 53 anos (40-70 anos), o início mediano de 56 dias após exposição (1-360 dias) e o tempo mediano de recuperação de 17,5 dias (1-42 dias). A descontinuação estava habitualmente associada à resolução dos sintomas.

Um estudo observacional de 2014 mostrou através de um modelo de regressão logística que os que tomavam estatinas era 2,4 vezes mais propensos a obter um resultado anormal (<24) no MMSE, comparado com os que não tomavam. ${ }^{14}$ Este modelo apresentava uma boa precisão de previsão (75\%). No entanto, a natureza observacional do estudo não permite estabelecer uma relação causal.

Um artigo de opinião de 2014, com um autorrelato da experiência com a toma de sinvastatina e pravastatina, refere aparecimento de sintomas associados ao início da toma de ambos os fármacos e desaparecimento dos mesmos com a suspensão de cada um deles. ${ }^{15} \mathrm{O}$ autor refere que como as alterações cognitivas são graduais, os pacientes podem não se aperceber delas a menos que sejam alertados.

Numa revisão de 2013, uma MA não mostrou associação estatisticamente significativa entre o uso de estatinas e alterações cognitivas em indivíduos saudáveis, três revisões revelam que os efeitos laterais cognitivos são raros e que a evidência é insuficiente para provar causalidade com demência ou doença de Alzheimer; que não há associação com o tipo ou dose das estatinas apesar de se sugerir diferença entre as hidrofílicas e lipofílicas; e uma delas mostra que as alterações cognitivas com a sinvastatina são raras e potencialmente se resolvem com a substituição de estatinas lipofílicas por hidrofílicas. Adicionalmente, dois ECR revelaram diminuição da função cognitiva com a sinvastatina e não mostraram efeitos na função cognitiva com a lovastatina. ${ }^{16}$ 


\section{CONCLUSÕES}

Após a análise da evidência disponível verifica-se que esta permanece controversa e inconsistente: alguns estudos mostram alterações cognitivas, entre as quais perda de memória, enquanto outros sugerem um efeito protetor na prevenção da demência e vários estudos não mostram alterações na função cognitiva com a toma de estatinas (SORT B). Admite-se que estas alterações possam ocorrer como uma reação rara, que se resolve com a suspensão do fármaco. Vários estudos apresentavam uma população pequena, sem poder estatístico para avaliar os riscos. Uma das limitações da presente revisão prende-se com o facto de algumas revisões não descreverem ao pormenor a população dos estudos incluídos, pelo que poderão não cumprir na totalidade os critérios de inclusão e exclusão da presente revisão. Assim, são necessários estudos prospetivos controlados para avaliar os efeitos das várias estatinas a curto e longo prazo. Conclui-se também que a evidência não apoia a alteração das guidelines, mas defende que não sejam desvalorizados os possíveis efeitos laterais.

\section{REFERÊNCIAS BIBLIOGRÁFICAS}

1. Centro de Conferência de Faturas. Dados de consumos de medicamentos no mercado do Serviço Nacional de Saúde. Lisboa: INFARMED; 2017.

2. INFARMED. 3.7. Antidislipidémicos. In: INFARMED, editor. Prontuário terapêutico on-line [homepage]. Lisboa: INFARMED; 2016 [cited 2018 Jan 28]. Available from: http://app10.infarmed.pt/prontuario/frameprimeiracapitulos.html

3. Perlmutter D. Cérebro de farinha - A chocante verdade sobre o trigo, o glúten e o açúcar: os assassinos silenciosos do seu cérebro. Alfragide: Lua de Papel; 2017.

4. INFARMED. Sinvastatina 20mg. In: Infomed: base de dados de medicamentos [Internet]. Lisboa: INFARMED; 2018. Available from: https://extranet.infarmed.pt/INFOMED-fo/pesquisa-avancada.xhtml

5. Björkhem I, Meaney S. Brain cholesterol: long secret life behind a barrier. Arterioscler Thromb Vasc Biol. 2004;24(5):806-15.

6. Banach M, Rizzo M, Nikolic D, Howard G, Howard V, Mikhailidis D. Intensive LDL-cholesterol lowering therapy and neurocognitive function. Pharmacol Ther. 2017;170:181-91.
7. Swiger KJ, Manalac RJ, Blumenthal RS, Blaha MJ, Martin SS. Statins and cognition: a systematic review and meta-analysis of short- and longterm cognitive effects. Mayo Clin Proc. 2013;88(11):1213-21.

8. Mancini GB, Baker S, Bergeron J, Fitchett D, Frohlich J, Genest J, et al. Diagnosis, prevention, and management of statin adverse effects and intolerance: Canadian Consensus Working Group update (2016). Can J Cardiol. 2016;32(7 Suppl):s35-s65.

9. Barry AR, O'Neill DE, Graham MM. Primary prevention of cardiovascular disease in older adults. Can J Cardiol. 2016;32(9):1074-81.

10. Macedo AF, Taylor FC, Casas JP, Adler A, Prieto-Merino D, Ebrahim S. Unintended effects of statins from observational studies in the general population: systematic review and meta-analysis. BMC Med. 2014;12:51.

11. Šimić I, Reiner Ž. Adverse effects of statins: myths and reality. Curr Pharm Des. 2015;21(9):1220-6.

12. Rojas-Fernandez CH, Goldstein LB, Levey Al, Taylor BA, Bittner V, The National Lipid Association's Safety Task Force. An assessment by the Statin Cognitive Safety Task Force: 2014 update. J Clin Lipidol. 2014;8(3 Suppl):S5-16.

13. Tuccori M, Montagnani S, Mantarro S, Capogrosso-Sansone A, Ruggiero E, Saporiti A, et al. Neuropsychiatric adverse events associated with statins: epidemiology, pathophysiology, prevention and management. CNS Drugs. 2014;28(3):249-72.

14. Mandas A, Congiu MG, Abete C, Dessi S, Manconi PE, Musio M, et al. Cognitive decline and depressive symptoms in late-life are associated with statin use: evidence from a population-based study of Sardinian old people living in their own home. Neurol Res. 2014;36(3):247-54.

15. McDonagh J. Statin-related cognitive impairment in the real world: you'll live longer, but you might not like it. JAMA Intern Med. 2014;174(12):1889.

16. Mancini GB, Tashakkor AY, Baker S, Bergeron J, Fitchett D, Frohlich J, et al. Diagnosis, prevention, and management of statin adverse effects and intolerance: Canadian Working Group Consensus update. Can J Cardiol. 2013;29(12):1553-68.

\section{CONFLITO DE INTERESSES}

A autora declara não ter quaisquer conflitos de interesse.

\section{ENDEREÇO PARA CORRESPONDÊNCIA}

Tânia Gonçalves

E-mail: tania_ftg@hotmail.com

https://orcid.org/0000-0002-0386-8687

Recebido em 03-04-2018

Aceite para publicação em 22-06-2020 


\section{ABSTRACT}

\section{STATINS: AN EVIDENCE-BASED REVIEW OF THE EFFECTS ON COGNITIVE FUNCTION}

Objective: Statin consumption has increased over the years and associated cognitive dysfunction has been described. It is known that the human brain contains about $25 \%$ of the cholesterol present in the human body. The aim of this review is to evaluate the cognitive side effects of statins in adults taking this class of anti-dyslipidemic drugs.

Data sources: National Guideline Clearinghouse, NICE Guidelines Finder, Canadian Medical Association Practice Guidelines Infobase, The Cochrane Library, Bandolier, DARE, Clinical Evidence e PubMed.

Methods: A survey of articles published between January 2013 and January 2018, in Portuguese and English, was conducted, using the MeSH terms Hydroxymethylglutaryl-CoA Reductase Inhibitors and Cognitive Dysfunction. The Strength of Recommendation Taxonomy (SORT) scale was used to assign levels of evidence and recommendation strengths.

Results: A total of 58 articles were found, of which eight met the inclusion and exclusion criteria: two systematic reviews, four reviews, one observational study, and one opinion article. After reading these studies, contradictory results were found: some showed side effects of statins on cognition, others showed no difference when compared with placebo and others even suggested a protective effect on dementia. Several studies did not have statistical power to assess the risks.

Conclusion: Controlled prospective studies are needed to evaluate the effects of statins on short- and long-term cognitive function. The evidence does not support the change of the actual guidelines but argues that the complaints should not be devalued.

Keywords: Hydroxymethylglutaryl-CoA reductase inhibitors; Cognitive dysfunction. 